ISSN 1991-8631

Short Communication

http://indexmedicus.afro.who.int

\title{
Application des méthodes statistique et analytique à l'évaluation de la composition ionique des eaux usées urbaines de la ville de Nouakchott
}

\author{
Abdoulaye Demba N'DIAYE ${ }^{1 *}$, Mohamed Ould Sid'Ahmed Ould KANKOU ${ }^{2}$ \\ et Baidy LO ${ }^{1}$ \\ ${ }^{1}$ Laboratoire de Chimie de l'Eau, Institut National de Recherches en Santé Publique de Nouakchott, BP 690, \\ Tél. (222) 52531 75, Mauritanie. \\ ${ }^{2}$ Laboratoire de Chimie de l'Eau et Environnement, Faculté des Sciences et Techniques de l'Université de \\ Nouakchott-BP 5026, Tél. (222) 52513 82, Mauritanie. \\ *Auteur correspondant ; E-mail: abdouldemba@yahoo.fr
}

\section{RESUME}

L'étude de la composition ionique des eaux usées urbaines de la ville de Nouakchott s'est déroulée au cours du mois de mars et avril 2009. Les paramètres physico-chimiques étudiés sont la température, le $\mathrm{pH}$, la conductivité électrique, des ions chlorures, calcium, magnésium, sodium, potassium, ammonium, orthophosphates et sulfates. L'application de l'Analyse en Composantes Principales sur ces résultats nous renseigne sur l'existence d'une relation étroite entre la conductivité électrique et la composition ionique de ces eaux usées urbaines. La méthode conductimétrique nous permet d'estimer les contributions moyennes des ions étudiés par rapport à la conductivité électrique moyenne enregistrée au niveau de ces eaux usées urbaines. Les ions ayant les contributions les plus élevées sont les chlorures, sodium et ammonium et possèdent respectivement $45,3 \%, 14,6 \%$ et $17,7 \%$. Les ions calcium et magnésium ont des contributions moyennes respectives de $9,6 \%$ et de $5,7 \%$ par contre, les ions potassium, sulfates et orthophosphates ont des valeurs de contributions inférieures à $5 \%$.

(C) 2010 International Formulae Group. All rights reserved.

Mots clés : ACP, Conductimétrie, Nouakchott, Mauritanie.

\section{INTRODUCTION}

De nombreuses études ont été conduites dans le domaine des eaux usées urbaines de la ville de Nouakchott surtout au niveau du périmètre maraicher de Sebkha (Schneider et Gagneux, 1997 ; Ould Selmane et Azandosessi, 1998; Azandosessi et al., 1999).

La présente étude se propose d'une part de suivre l'évolution des paramètres physicochimiques des effluents de la ville de
Nouakchott et d'autre part d'évaluer la composition ionique de ces effluents avec des procédés statistiques et analytiques. Pour mieux évaluer cette composition ionique, nous avons utilisé deux méthodes: l'Analyse en Composante Principale (A.C.P) et la conductimètrie.

\section{MATERIEL ET METHODES \\ Milieu d'étude}

La ville de Nouakchott est une ville 
côtière, située entre $18^{\circ} 07$ de latitude Nord et $16^{\circ} 01$ de longitude Ouest. Nouakchott est alimenté en eau potable par le champ captant d'Idini, situé sur la route de l'espoir à environ $60 \mathrm{~km}$ de la ville (Mint El Bezeid, 2007). Le site du prélèvement est la STEP (Station de Traitement des Eaux Polluées) ou l'ensemble des eaux usées brutes drainées par certains quartiers et de quelques unités industrielles connectées aussi à la STEP (une Société de mise en bouteille et une société de pêche spécialisée dans le domaine de vente de céphalopodes poulpes et seiches). Le débit actuellement traité par la station est de 458 $\mathrm{m}^{3}$ /jour (AMEXTIPE, 2000).

\section{Méthodes d'analyses}

Les prélèvements ont été effectués hebdomadairement au niveau de la STEP au cours des mois de mars et avril 2009. Les analyses ont eu lieu immédiatement après prélèvement au laboratoire de chimie de l'eau de l'INRSP (Institut National de Recherches en Santé Publique) à Nouakchott. Les paramètres physicochimiques étudiés sont: la température, le $\mathrm{pH}$, la conductivité, les chlorures, calcium, magnésium, sodium, potassium, ammonium, orthophosphates et sulfates.

Le $\mathrm{pH}$ et la température ont été déterminés par un pH-mètre de type Hanna muni d'une sonde mesurant la température. La conductivité a été mesurée par un conductimètre de type Hanna. Les chlorures sont mesurés par la méthode de Mohr en présence de nitrate d'argent. Les ions calcium et le magnésium sont dosés par complexométrie à l'aide d'une solution de sel disodique d'acide éthylenediamine tétracétique (EDTA) (Rodier, 1996). Les ions ammonium, ortho phosphates et sulfates sont analysés par des méthodes colorimétriques à l'aide d'un spectrophotomètre UV Visible de type $722 \mathrm{~S}$ Beijing. Les ions ammonium par le réactif Nessler à une longueur d'onde de 420 nm. Pour le dosage des ortho phosphates, on utilise un réactif molybdique susceptible d'un dosage colorimétrique à une longueur d'onde de $700 \mathrm{~nm}$. Les ions sulfates sont précipités en milieu aqueux par le baryum en présence de thymol et de gélatine à une longueur d'onde de $530 \mathrm{~nm}$. Pour les ions sodium et potassium, nous avons utilisé un photomètre à émission atomique à flamme de type Corning. L'étude statistique a été basée sur l'ACP. Les matrices de corrélations intermédiaires ont été obtenues avec un logiciel XLSTAT 2010. La conductimètrie permet de mesurer les propriétés conductrices d'une solution. La valeur de la conductivité CE peut être calculée à partir des conductivités molaires ioniques $\lambda_{\mathrm{i}}$ des ions qui composent cette solution, ainsi que de leurs concentrations $\left[\mathrm{X}_{\mathrm{i}}\right]: \mathrm{CE}=\Sigma \lambda_{\mathrm{i}}$ $* \mathrm{Z}_{\mathrm{i} *}\left[\mathrm{X}_{\mathrm{i}}\right]$ avec CE : Conductivité Electrique en $\mathrm{mS} . \mathrm{m}^{-1}, \lambda_{\mathrm{i}}$ en $\mathrm{S} . \mathrm{m}^{2} . \mathrm{mol}^{-1}, \mathrm{z}_{\mathrm{i}}$ est la charge électrique d'un ion et $\left[\mathrm{X}_{\mathrm{i}}\right]$ en mol.m ${ }^{-3}$ (Bonté et al., 2008).

\section{RESULTATS}

\section{Aspect qualitatif des eaux usées urbaines} de la ville de Nouakchott

Les Figures 1, 2 et 3 donnent les évolutions hebdomadaires de la température, du $\mathrm{pH}$ et de la conductivité électrique au cours des mois de mars et avril 2009. Le Tableau 1 donne les résultats des ions chlorures, calcium, magnésium, sodium, potassium, ammonium, orthophosphates et sulfates au cours du mois de mars et avril 2009.

\section{Application de l'analyse en composantes principales}

Le traitement des données par l'analyse en composantes principales, en utilisant comme variables la conductivité, les ions chlorures, calcium, magnésium, sodium, potassium, ammonium, orthophosphates et sulfates, et comme individus les 27 prélèvements effectués au cours du mois de mars et d'avril 2009. Le Tableau 2 donne les matrices de corrélations intermédiaires. 
Application de la conductimétrie: détermination des contributions des ions à la conductivité totale

Les variations de la conductivité électrique des eaux usées urbaines de la ville de Nouakchott ont été suivies hebdomadairement au cours du mois de mars et d'avril 2009. On a également dosé sur les échantillons les principaux ions (chlorures, sodium, potassium, calcium, magnésium, sulfates, orthophosphates et ammonium). Les constantes des ions étudiés tirées des travaux de Bonté et al. (2008) sur la relation entre conductivité et la composition ionique des eaux usées urbaines. Le Tableau 3 donne les contributions moyennes des ions étudiés par rapport à la conductivité moyenne enregistrée au niveau des eaux usées urbaines de Nouakchott.

Tableau 1 : Résultats de l'évolution des ions étudiés.

\begin{tabular}{llllll}
\hline Paramètres & Unités & Max. & Min. & Moy. & Ecart Types \\
\hline $\mathrm{Cl}^{-}$ & mg/litre & 1633 & 397,6 & 954,4 & 333,64 \\
$\mathrm{Na}^{+}$ & mg/litre & 390 & 191 & 290,7 & 70,18 \\
$\mathrm{Ca}^{2+}$ & mg/litre & 218,04 & 67,11 & 133,23 & 63,98 \\
$\mathrm{Mg}^{2+}$ & mg/litre & 94,28 & 11,10 & 58,8 & 32,95 \\
$\mathrm{NH}_{4}{ }^{+}$ & mg/litre & 292,05 & 119,14 & 212,5 & 61,86 \\
$\mathrm{PO}_{4}{ }^{3-}$ & mg/litre & 39,15 & 22,78 & 28,16 & 4,35 \\
$\mathrm{~K}^{+}$ & mg/litre & 39 & 26 & 31,83 & 4,41 \\
$\mathrm{SO}_{4}{ }^{2-}$ & mg/litre & 53,29 & 30,64 & 43,85 & 6,54 \\
\hline
\end{tabular}

Tableau 2 : Matrice des corrélations inter-élémentaires.

\begin{tabular}{lrrrrrrrrr}
\hline Variables & \multicolumn{1}{c}{$\mathbf{C E}$} & $\mathbf{C l}^{-}$ & $\mathbf{N H}_{4}^{+}$ & $\mathbf{P O}_{\mathbf{4}}{ }^{3-}$ & $\mathbf{S O}_{\mathbf{4}}{ }^{2-}$ & $\mathbf{N a}^{+}$ & $\mathbf{K}^{+}$ & $\mathbf{C a}^{2+}$ & $\mathbf{M g}^{2+}$ \\
\hline $\mathbf{C E}$ & $\mathbf{1}$ & & & & & & & & \\
$\mathbf{C l}$ & 0,586 & $\mathbf{1}$ & & & & & & & \\
$\mathbf{N H}_{\mathbf{4}}{ }^{+}$ & 0,510 & 0,033 & $\mathbf{1}$ & & & & & & \\
$\mathbf{P O}_{\mathbf{4}}{ }^{3-}$ & $-0,041$ & $-0,827$ & 0,204 & $\mathbf{1}$ & & & & & \\
$\mathbf{S O}_{\mathbf{4}}{ }^{2-}$ & 0,554 & $-0,074$ & $-0,090$ & 0,550 & $\mathbf{1}$ & & & & \\
$\mathbf{N a}^{+}$ & 0,950 & 0,323 & 0,481 & 0,262 & 0,751 & $\mathbf{1}$ & & \\
$\mathbf{K}^{+}$ & $\mathbf{0 , 9 5 1}$ & 0,307 & 0,594 & 0,265 & 0,672 & $\mathbf{0 , 9 9 1}$ & $\mathbf{1}$ & & \\
$\mathbf{C a}^{2+}$ & 0,932 & 0,514 & 0,203 & 0,047 & 0,777 & 0,941 & 0,896 & $\mathbf{1}$ & \\
$\mathbf{M g}^{2+}$ & 0,946 & 0,500 & 0,251 & 0,068 & 0,771 & $\mathbf{0 , 9 5 7}$ & 0,917 & $\mathbf{0 , 9 9 9}$ & $\mathbf{1}$ \\
\hline \multicolumn{2}{c}{ Corrélations significatives $(\mathrm{p}<0,05)$ en gras et CE : Conductivité électrique. } & & &
\end{tabular}

Tableau 3 : Contributions des différents ions à la conductivité totale.

\begin{tabular}{lcccccccc}
\hline Ions & $\mathbf{C l}^{-}$ & $\mathbf{N a}^{+}$ & $\mathbf{K}^{+}$ & $\mathbf{C a}^{2+}$ & $\mathbf{M g}^{2+}$ & $\mathbf{S O}_{\mathbf{4}}{ }^{2-}$ & $\mathbf{P O}_{\mathbf{4}}{ }^{3-}$ & $\mathbf{N H}_{\mathbf{4}}{ }^{+}$ \\
\hline $\mathbf{Z}$ & 1 & 1 & 1 & 2 & 2 & 2 & 3 & 1 \\
$\boldsymbol{\lambda}\left(\mathbf{m . m} \mathbf{m}^{\mathbf{2}} / \mathbf{m o l e}\right)$ & 7,63 & 5,01 & 7,35 & 6,95 & 5,31 & 8 & 9,28 & 7,34 \\
$(\boldsymbol{\%})$ & 45,3 & 14,6 & 1,7 & 9,6 & 5,7 & 2 & 2,2 & 17,8 \\
\hline
\end{tabular}




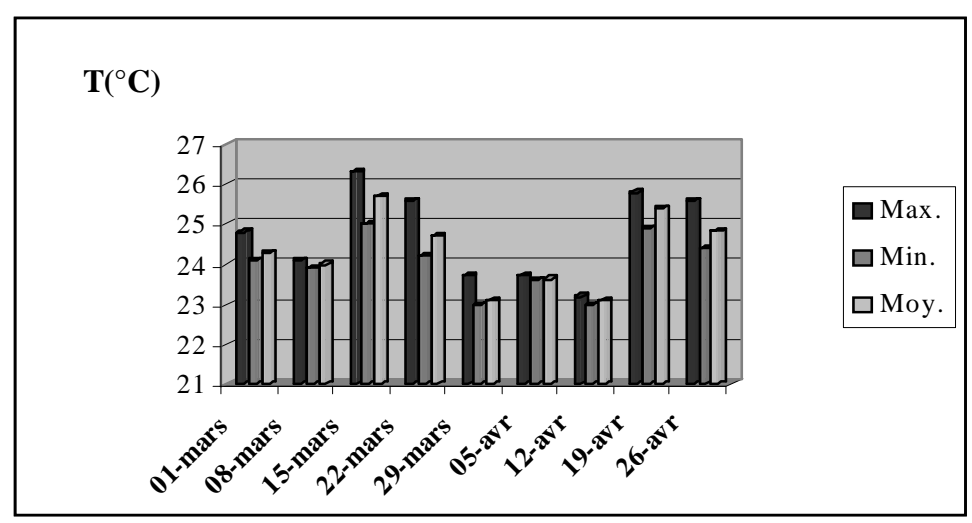

Figure 1 : Evolution de la température.

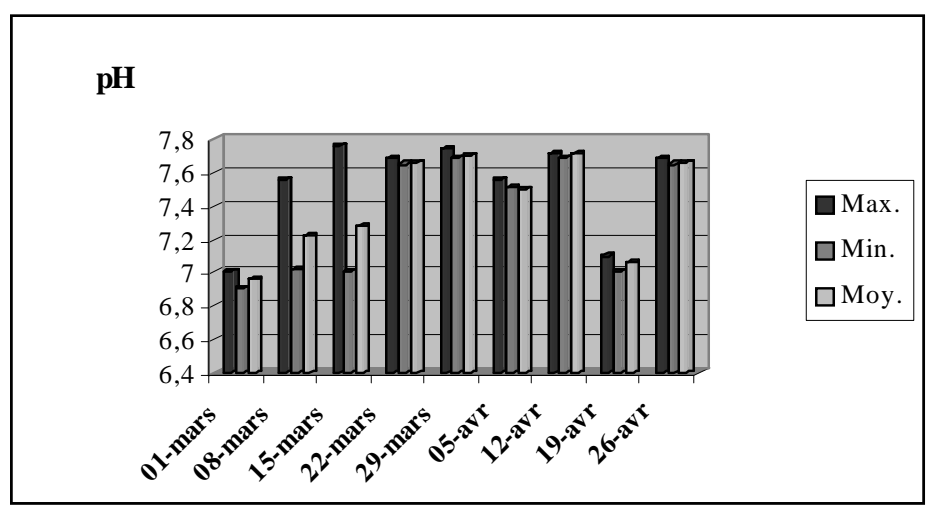

Figure 2 : Evolution du $\mathrm{pH}$.

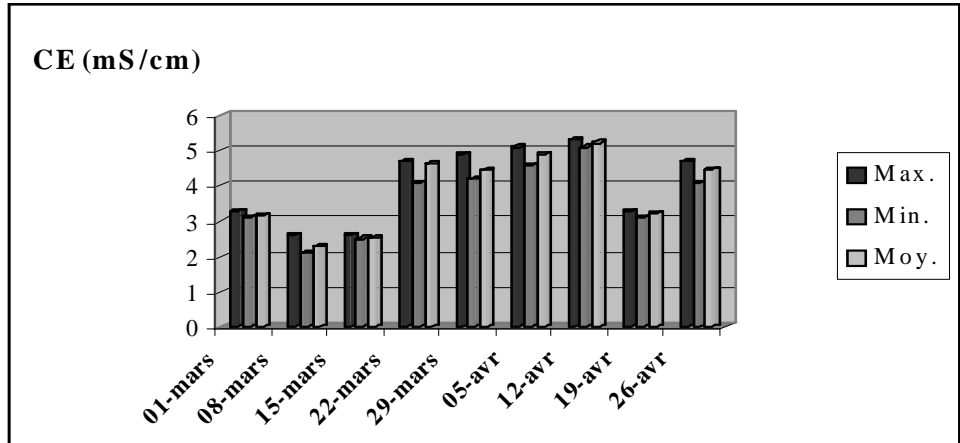

Figure 3 : Evolution de la conductivité.

\section{DISCUSSION}

Les températures enregistrées au niveau des eaux usées urbaines de Nouakchott varient de $23{ }^{\circ} \mathrm{C}$ à $26,3{ }^{\circ} \mathrm{C}$ (Figure 1). Les températures enregistrées sont inférieures à 30
${ }^{\circ} \mathrm{C}$ (Journal officiel de la République Algérienne, 2003) et $35^{\circ} \mathrm{C}$ considérée comme valeur limite de rejet direct dans le milieu récepteur (Ministère de l'Environnement du Maroc, 2002). Le pH oscille entre 6,91 et 7,76 
(Figure 2). La conductivité électrique est probablement l'une des plus simples et des plus importantes pour le contrôle de la qualité des eaux usées. Elle traduit le degré de minéralisation globale, elle nous renseigne sur le taux de salinité (El Guamri et Belghyti, 2006; El Guamri et Belghyti, 2007). La valeur maximale de la conductivité enregistrée au niveau des eaux usées urbaines de la ville de Nouakchott est de $5,3 \mathrm{mS} / \mathrm{cm}$ et la valeur minimale est de $2,1 \mathrm{mS} / \mathrm{cm}$ (Figure 3 ). Ces résultats pourraient être expliqués par le rejet des eaux usées résiduaires des unités industrielles connectées à la STEP. Les valeurs de la conductivité enregistrées au niveau de l'effluent de la STEP dépassent 2 $\mathrm{mS} / \mathrm{cm}$ (Journal Officiel de la République Algérienne, 2003). La valeur maximale en chlorures atteinte enregistrée au niveau des eaux usées urbaines de la ville de Nouakchott est de $1633 \mathrm{mg} /$ litre et la valeur minimale est de 397,6 mg/litre (Tableau 1). Les concentrations enregistrées dépassent largement la concentration limite de rejet direct dans le milieu récepteur $(50 \mathrm{mg} / \mathrm{litre})$ (Ministère de l'Environnement du Maroc, 2002). Les concentrations maximales et minimales en sodium obtenues au niveau des eaux usées urbaines de la ville de Nouakchott sont respectivement de $390 \mathrm{mg} /$ litre et de 191 mg /litre (Tableau 1). Les teneurs en calcium et en magnésium enregistrées varient respectivement de $67,11 \mathrm{mg} /$ litre à 226,05 mg /litre (Tableau 1) et 11,10 mg /litre à 94,28 mg /litre (Tableau 1). L'azote ammoniacal peut provenir de ruissellements urbains. L'urine est la principale source d'ammonium dans les eaux usées domestiques (Bonté et al, 2008). $\mathrm{La}$ valeur moyenne maximale atteinte en ammonium est de 292,05 mg/litre et la valeur moyenne minimale est de $119,14 \mathrm{mg} /$ litre (Tableau 1). La grande partie du phosphore organique provient également des déchets du métabolisme des protéines et de son élimination sous forme de phosphates dans les urines par l'homme (Du Chaufour, 1997). Les teneurs en orthophosphates oscillant entre 20,78 mg/litre et 39,15 mg /litre (Tableau 1). Ces teneurs en orthophosphates sont supérieures à $10 \mathrm{mg}$ /litre considéré comme étant une valeur limite acceptable d'un rejet direct dans le milieu récepteur (Ministère de l'Environnement du Maroc, 2002). La concentration maximale en potassium enregistrée des eaux usées urbaines de la ville de Nouakchott est de $39 \mathrm{mg} /$ litre et la concentration minimale est de $21 \mathrm{mg} /$ litre (Tableau 1). Les concentrations en sulfates obtenues au niveau des eaux usées urbaines de Nouakchott oscillent entre $30,64 \mathrm{mg}$ /litre et 53,29 mg /litre (Tableau 1).

L'ACP est un outil d'analyse de données qui permet d'expliquer la structure des corrélations ou des covariances en utilisant des combinaisons linéaires des données originelles (Lagarde, 1995 ; Maliki, 2000). Des corrélations positives très significatives ont été observées entre la conductivité électrique et les ions calcium, magnésium, sodium et potassium (Tableau 2). Des corrélations positives moyennement significatives ont été observées entre la conductivité électrique et les ions chlorures, ammonium et sulfates (Tableau 2). Une corrélation négative et faiblement significative a été observée entre la conductivité électrique et les ions orthophosphates (Tableau 2). D'après la conductimétrie, les ions ayant les contributions moyennes les plus élevées sont les chlorures, sodium et ammonium et possèdent respectivement $45,3 \%, 14,6 \%$ et $17,7 \%$ (Tableau 3). Les contributions moyennes en calcium et en magnésium sont respectivement de $9,6 \%$ et $5,7 \%$ (Tableau 3 ). Les ions potassium, sulfates et orthophosphates ont des valeurs de contributions inférieures à $5 \%$. Les valeurs moyennes de contribution en potassium, sulfates et en orthophosphates sont respectivement $1,7 \%, 2 \%$ et $2,2 \%$ (Tableau 3). 


\section{Conclusion}

L'étude de la composition ionique des eaux usées urbaines de la ville de Nouakchott s'est déroulée au cours du mois de mars et d'avril 2009. Les paramètres physicochimiques étudiés sont la température, le $\mathrm{pH}$, la conductivité électrique, des ions chlorures, calcium, magnésium, sodium, potassium, ammonium, orthophosphates et sulfates. L'application de l'ACP sur ces résultats nous renseigne sur l'existence d'une relation étroite entre la conductivité électrique et la composition ionique des eaux usées urbaines de la ville de Nouakchott. La méthode conductimétrique nous permet d'estimer les contributions des ions étudiés à la conductivité des effluents.

\section{REFERENCES}

AMEXTIPE. 2000. Etude sur la Stratégie de Développement des villes de Nouakchott, Nouadhibou et Kaédi (Volet: Evaluation Environnementale et Sociale Stratégique), Rapport préliminaire A 2003, République Islamique de Mauritanie.

Azandossessi A, Ould Selmane ML, Ould Baba L, Benzeroug EH, Cissé G, Tanner M. 1999. Projet de préservation de l'unique espace vert de Nouakchott : le site de Sebkha « Nouakchott El Khadra ». Document de projet. OMS, Nouakchott.

Bonté SL, Pons M, Potier O, Rocklin P. 2008. Relation between conductivity and ion content in urban wastewater. Journal of Water Science, 21(4): 429- 438.

Du Chaufour P. 1997. Abrégé de Pédologie: Sol, Végétation et Environnement ( $5^{\text {ème }}$ édn). Masson.

El Guamri Y, Belghyti D. 2006. Etude de la qualité physico-chimique des eaux usées brutes de la commune urbaine de Saknia, rejetées dans le lac Fouarat (Kénitra, Maroc). Journal Africain des Sciences de l'Environnement, 1: 53-60.

EL Guamri Y, Belghyti D. 2007. "Etude de l'aspect physico-chimique et parasitologique des eaux usées destinées à l'irrigation de périmètre péri-urbain de Fouarat (Kénitra, Maroc)". Revue Agronomie Africaine, 19(3): 251-261.

Journal Officiel de la République Algérienne, 1993. Normes de rejets dans le milieu récepteur. Journal Officiel de la République Algérienne, 46: 7-12.

Lagarde J. 1995. Initiation à l'Analyse des Données. Ed. Dunod : Paris ; 157 p.

Maliki AM. 2000. Etude hydrologique hydrochimique et isotopique de la nappe profonde de Sfax (Tunisie). Thèse de Doctorat Fac. Sci. Sfax, 301 p.

Ministère de l'Environnement du Maroc. 2002. « Normes marocaines, Bulletin officiel du Maroc », $\mathrm{N}^{\circ} 5062$ du 30 ramadan 1423. Rabat.

Mint El Bezeid F. 2007. Evaluation de risques Environnementaux qui menacent la zone côtière de Nouakchott et les solutions possibles (Mauritanie). Mémoire DESA Faculté des Sciences, EL Jadida Université Chouaib Doukkali Maroc.

Ould Selmane ML, Azandosessi A.1997. Méthode accélérée de planification participative. Etude de cas : sites maraîchers de Tar El Zatar (Moughataa de Dar Naim) et de Sebkha. Rapport de recherche. Projet de recherche «Epidémio REU », OMS, Nouakchott.

Rodier J. 1996. L'Analyse de l'Eau Naturelle, Eaux Résiduaires, Eau de Mer (8 éme édn). Denod : Paris ; 1383 p.

Schneider C, Gagneux S. 1997. Impact sanitaire de l'utilisation d'eaux usées et polluées en agriculture urbaine: Cas du maraîchage à Nouakchott, République. Islamique de Mauritanie. Travail de Diplôme, Institut Tropical Suisse, Université de Bâle. 